\title{
Kalorimetrische Faecesuntersuchungen.
}

\author{
Von \\ Dr. Hans Lohrisch. \\ (Aus der I. inneren Abteilung des Friedrichstädter Krankenhauses zu Dresden. \\ Oberarzt Prof. Dr. Ad. Schmidt.) \\ (Der Redaktion zugegangen am 9. März 1904.)
}

Die direkte kalorimetrische Brennwertsbestimmung organischer und anorganischer Substanzen ist eine physikalische Untersuchungsmethode, welche bisher vorwiegend dazu benutzt wurde, die Brennwerte von Nahrungsmitteln und Heizmaterialien festzustellen. Die Bestimmungen werden mit Hilfe des Kalorimeters ausgeführt, welcher nach folgendem Prinzipe arbeitet: Die zu untersuchende Substanz wird unter einem Überdruck und Überschuß von Sauerstoff im luftleeren Raume verbrannt. Es wird dadurch erreicht, daß alle Elemente so hoch wie möglich oxydiert werden, sodaß die gesamte latente Energie des betreffenden Stoffes in Wärme übergeführt wird. Damit die entwickelte Wärme sich nicht im Raume verliert, wird sie gezwungen, sich in einem bestimmten Medium, nämlich in Wasser, auszubreiten. Die Erwärmung des Wassers wird direkt thermometrisch gemessen und daraus die der Wärmeentwicklung entsprechende Kalorienmenge berechnet.

Das Kalorimeter ist erst seit dem Jahre 1877 durch Stohmann ${ }^{1)}$ bekannter geworden. Zu der Zeit, als Stohmann anfing, sich mit kalorimetrischen Untersuchungen $\mathrm{zu}$ beschäftigen, existierte nur eine einzige diesen Gegenstand behandelnde Arbeit von Frankland ${ }^{2}$ ) aus dem Jahre 1866. Dieser arbeitete mit einem von Lewis Thompson für die Brennwertsbestimmung organischer Substanzen angegebenen Kalorimeter: Die Substanzen wurden im geeigneten Verhältnis mit Kaliumchlorat und Braunstein gemischt und in einer Kupferpatrone entzündet; die bei der Verbrennung entwickelten Gase durchströmten das Wasser des Kalori-

1) Über den Wärmewert der Bestandteile der Nahrungsmittel. Zeitschrift für Biologie. Neue Folge, Bd. 13, 1895.

2) Philos. Mag., Bd. 32, S. 182 (cit. nach Stohmann l. c.) 
meters und gaben dabei ihre Wärme an dieses ab. Stohmann ${ }^{1}$ ) benutzte diesen Apparat seit 1877, fand aber an der Methode wie am Apparat eine Anzahl Fehler, sodaß genaue Resultate nicht zu erzielen waren. Erst im Jahre 1884 war der Apparat nach Überwindung zahlreicher Schwierigkeiten soweit vervollkommnet, daß genaue Brennwertsbestimmungen ermöglicht wurden. Eine wesentliche Modifikation von Stohmann war die, daß er die Kupferpatronen durch solche aus Platin ersetzte, wodurch eine beträchtliche Fehlerquelle ausgeschaltet wurde. Rubner ${ }^{2}$ ) konnte später die Korrekturen Stohmanns an dem Franklandschen Apparate vollkommen bestätigen. Unter den von ihm angebrachten Neuerungen ist die wichtigste die Einführung von Glashülsen an Stelle der Kupfer- und Platinpatronen.

Hochbedeutsam für die Weiterentwicklung der Kalorimetrie war die Erfindung der Berthelotschen Bombe im Jahre 1886, deren genaue Beschreibung sich bei Langbein ${ }^{3}$ ) findet. Diese Bombe besteht im wesentlichen aus einem mit Platin ausgekleideten Gußstahltiegel, in dem die Verbrennung vor sich geht. Zur Entwicklung von Sauerstoff wird aber nicht mehr Kaliumchlorat verwendet, sondern es wird Sauerstoff direkt von außen unter einem Druck von 25 Atmosphären eingeleitet. Der Berthelotsche Apparat ermöglicht Brennwertsbestimmungen von staunenswerter Genauigkeit. Es wurde denn auch die alte Kaliumchloratmethode alsbald aufgegeben und allgemein Berthelots Bombe benutzt.

Im Laufe der Zeit erfuhr die Berthelotsche Methode allerlei Verbesserungen, so von Stohmann, Kleber und Langbein ${ }^{4}$ ) und insbesondere von Hempel. ${ }^{5}$ ) Von letzterem wurde zur Bestimmung des Heizwertes von Brennstoffen ein Kalorimeter konstruiert, das im Prinzip der Berthelotschen Bombe entspricht. Es ist aber in kleineren Dimensionen gehalten, bedeutend billiger. (Tiegel aus Flußeisen mit Emailleauskleidung) und eignet sich auch für die Verbrennung staubförmiger Substanzen, die im Berthelotschen Apparat nicht möglich war.

Trotzdem die Kalorimetrie im Laufe der Jahre in so vollkommener Weise ausgearbeitet worden ist, hat sie sich bisher unter den der wissenschaftlichen Medizin dienenden Untersuchungsmethoden keinen festen Platz erobern können. Und doch scheint es, als ob die Kalorimetrie auch für theoretische

i) $1 . \mathrm{c.}$.

2) Kalorimetrische Untersuchungen. Zeitschrift für Biologie. Neue Folge, 3. Bd., 1885.

3) Chemische und kalorimetrische Untersuchung von Brennstoffen. Zeitschrift für angewandte Chemie, 1900, S. 1227 u. 1259.

4) Journal für prakt. Chemie, Bd. 39, 1889.

5) Gasanalytische Methoden. Braunschweig 1890. 
und praktische klinische Zwecke ein überaus wertvolles Hilfsmittel sein müßte. Es geht dies besonders aus den Mitteilungen von Schloßmann ${ }^{1}$ ) hervor.

Schloßmann hat gelegentlich kalorimetrischer Untersuchungen von Säuglingskoten und Harn gefunden, daß die Kalorimetrie außerordentlich brauchbar ist, wenn es sich darum handelt, Untersuchungen über den Kraftwechsel anzustellen, d. h. die mit der Nahrung eingeführte Energie mit den Ausgaben an Kraftvorrat, die den Organismus im Harn und Kot verlassen, zu vergleichen. Er konnte sich aus dem Vergleich der Brennwerte der Nahrung und des Kotes auch sehr gut ein Urteil darüber bilden, ob die Ernährung der Säuglinge eine rationelle war und ob der Darm genügend funktionierte oder nicht. Schloßmann hält die kalorimetrische Untersuchungsmethode für "die beste Funktionsprüfung des Verdauungsapparates».

Es würde danach die Kalorimetrie für klinische Zwecke hauptsächlich in Frage kommen für die Ausführung von Untersuchungen des Gesamtkraftwechsels und der Ausnutzung von Nahrungsmitteln. Derartige Untersuchungen lösen bisher ihre Aufgabe auf dem Wege der chemischen Analyse, d. h. es werden Stickstoff, Fett und Kohlehydrate in der Nahrung und in den Entleerungen bestimmt und miteinander verglichen. Wenn man bedenkt, wie zahlreich, umständlich und zeitraubend die bei derartigen Versuchen nötigen Einzeluntersuchungen sind, so würde es höchst willkommen sein, wenn die direkten Brennwertsbestimmungen solche Untersuchungen abkürzen und vereinfachen könnten. Ich möchte hierbei nicht unerwähnt lassen, daß man in neuester Zeit versucht hat, die zahlreichen chemischen Untersuchungen bei Stoffwechselversuchen durch die kalorimetrische Messung zu ersetzen, wie es scheint mit bestem Erfolg. Atwater ${ }^{2}$ ) hat ein «Respirationskalorimeter» konstruiert, in welchem die $\mathrm{CO}_{2}$ - und $\mathrm{H}_{2} \mathrm{O}$-Abgabe gemessen werden kann.

1) Über die Bedeutung kalorimetrischer Untersuchungen für klinische Zwecke. Berl. klin. Wochenschrift 1903, Nr. 12.

2) Neuere Arbeiten über Stoff- und Kraftbilanz des menschlichen Körpers von Zuntz, Berlin. Sammelreferat nach Bull. Nr. 21, 44, 63, 69 und 109 d. U. St. Dep. of Agric., Washington. 
Gleichzeitig ist es möglich, die gesamte von der Versuchsperson abgegebene Wärme direkt zu messen und zwar in folgender Weise: Die abgegebene Wärme wird von einem den Respirationsraum durchsetzenden Wasserstrom, der in einem Röhrensystem im Innern des Apparates fließt, aufgenommen. Dieser Wärmeapparat ist durch drei Wände, die zwei Luftschichten einschließen, gegen die Umgebung thermisch isoliert. In den Luftschichten sind Thermoelemente angebracht, die $\mathrm{zu}$ einem Galvanometer hinleiten. Es wird auf diese Weise die gesamte abgegebene Wärme direkt kalorimetrisch gemessen. Diese Messungen wurden durch Stoffwechselversuche, die in der gewöhnlichen Weise ausgeführt wurden, kontrolliert und dabei eine sehr befriedigende Übereinstimmung zwischen Verbrennungswärme der umgesetzten Stoffe und der gesamten direkt gemessenen Wärmeabgabe erzielt.

Ehe man nun bei Kraftwechseluntersuchungen und bei Ausnutzungsversuchen die chemische Untersuchung des Kotes durch das kalorimetrische Verfahren ersetzen kann, muß man sich darüber vergewissern, in welchem Verhältnis die direkt kalorimetrisch ermittelten Brennwerte zu den durch die Elementaranalyse gefundenen Kalorienzahlen stehen, ob wesentliche Differenzen zwischen beiden bestehen, die eventuell $\mathrm{zu}$ Fehlern bei der Berechnung führen könnten, und welche sonstigen Aufschlüsse die kalorimetrische Untersuchung der Faeces des erwachsenen, gemischte Kost genießenden Menschen geben kann, zumal im Hinblick auf Schloßmanns ${ }^{1}$ ) Untersuchungen an Säuglingen. Diesem Zweck dienen die folgenden kalorimetrischen Faecesuntersuchungen, die ich auf Veranlassung des Herrn Prof. Dr. Schmidt ausgeführt habe. Die betreffenden Kote hat Herr Prof. Schmidt zum Teil selbst gesammelt und analysiert und mir zur Verfügung gestellt (in der Tabelle die Fälle 1, 2, 3, 6, 7, 8, 9, 10, 11, 12). Eine kleine Anzahl von. Koten wurden von mir gesammelt und chemisch untersucht. Sämtliche Kote sind mittels der Probediät von Schmidt und Strasburger ${ }^{2}$ ) gewonnen und zwar beziehen sie sich immer

1) $1 . \mathrm{c}$.

2) Die Faeces des Menschen im normalen und krankhaften $\mathrm{Zu}$ stande. I. Teil. Berlin 1902. 
auf einen dreitägigen Gebrauch derselben. Die Abgrenzung der Faeces geschah mit Karmin. Von jedem Kote sind die Werte für Stickstoff, Fett und Kohlehydrate durch Analyse ermittelt: die Stickstoffzahlen nach Kjeldahl, die Fette durch dreitägige Extraktion im Soxhletapparat nach vorheriger Spaltung mit salzsaurem Alkohol, die Kohlehydrate nach der Methode von Strasburger ${ }^{1}$ ) unter Anwendung der Kupferrhodanürmethode von Volhard-Pflüger. Sodann wurden von der Probediät und von sämtlichen Koten die Brennwerte direkt mit Hilfe des Hempelschen Kalorimeters bestimmt. Herr Prof. Schloßmann hatte die Güte, mir sein im Dresdner Säuglingsheim aufgestelltes Kalorimeter zur Verfügung zu stellen, wofür ich ihm meinen verbindlichsten Dank ausspreche.

Ich lasse im folgenden eine kurze Beschreibung des Hempelschen Kalorimeters und seiner Anwendung zur Verbrennung von Koten folgen. Das Kalorimeter von Hempel enthält folgende Teile:

1. Einen Preßapparat, in dem die zu untersuchenden fein pulverisierten Brennstoffe zu Blöckchen gepreßt werden.

2. Eine Autoklave aus Flußeisen von ca. $250 \mathrm{ccm}$ Inhalt, welche auf einen Druck von 25 Atmosphären geprüft ist. Der Verschluß der Autoklave wird durch ein aufschraubbares Kopfstück gebildet. Dieses trägt ein Schraubenventil, einen Flanschenrohransatz und 2 Elektroden. Am untern Ende des Kopfstückes sind zwei Eisenstäbe angebracht, welche an ihren Enden je eine Platinöse tragen und in die Autoklave hineinragen. An den Platinösen wird der zu verbrennende Block mittels Platindrahtes befestigt.

3. Einen Sauerstoffentwickler mit Manometer, welcher an dem Flanschenrohransatz anzuschrauben ist. (Im Säuglingsheim ein Eisenzylinder mit komprimiertem Sauerstoff, an dem sich der Druck durch ein Manometer regulieren läßt.)

4. Das eigentliche Kalorimeter. Dieses besteht aus einem Metallgefä $b$, welches das Kalorimeterwasser enthält und in einem Abstand von ca. $2 \mathrm{~cm}$ in einem Holzgefäß aufgehängt ist. In das Metallgefäß wird die Autoklave eingesetzt, ferner ein feines Thermometer und eine Rührvorrichtung. Das Ganze wird durch einen Deckel abgeschlossen, welcher Öffnungen für das Thermometer, das Rührwerk und die Elektroden hat.

Eine Brennwertshestimmung mit dem Hempelschen Kalorimeter gestaltet sich in praxi folgendermaßen:

Der getrocknete Kot wird möglichst fein verrieben und nochmals bei ca. $90^{\circ}$ im Trockenschrank, bei sehr fettreichen Stühlen im Exsiccator

1) Schmidt und Strasburger 1. c. II. Teil. 
getrocknet. Ca. $1 \mathrm{~g}$ des völlig trockenen Pulvers wird mit Hilfe des Preßapparates zu einem Blöckchen gepreßt. Gleichzeitig wird ein $11 \mathrm{~cm}$ langer Zwirnsfaden, dessen Brennwert bekannt ist, mit in die Substanz hineingedrückt. Das Blöckchen wird genau gewogen. Nun wird das Blöckchen an einem 0,1 mm dicken Platindraht, der zwischen den am Kopfstück der Autoklave befindlichen beiden Platinösen ausgespannt wird, mit dem einen Ende des Zwirnsfadens aufgehängt. Der Block schwebt jetzt in einem kleinen Platintiegel, der in ein an den beiden Platinösen aufgehängtes Tonschälchen eingesetzt ist. Durch den Platintiegel werden etwa abbröckelnde Teilchen des Blockes aufgesammelt und so der Verbrennung zugänglich gemacht. Die Autoklave wird hierauf fest verschlossen.

Aus dem Sauerstoff behälter, der an den Flanschenrohransatz luftdicht angeschraubt wird, wird zunächst durch einströmenden Sauerstoff die in der Autoklave noch vorhandene Luft verdrängt, sodann wird die Autoklave bei einem Druck von 20-21 Atmosphären unter Kontrolle des Manometers mit Sauerstoff gefüllt und durch das Schraubenventil abgeschlossen. Nunmehr wird die Autoklave in das Metallgefäß des Kalorimeters eingesetzt, welches genau 1 Liter Aqua dest. enthält. Die Temperatur dieses Wassers ist um ca. $1,5^{\circ}$ C. kälter als die umgebende Luft. Man erreicht dies durch vorherige Mischung des Wassers.

Nachdem man noch das Rührwerk in das Metallgefäß gebracht hat, wird der Apparat durch den Deckel, welcher das Thermometer und 2 Elektroden trägt, geschlossen. Die Elektroden werden mit einem kleinen zweizelligen Akkumulator verbunden. Es beginnt jetzt die eigentliche Verbrennung, welche nur noch kurze Zeit in Anspruch nimmt. Mittels der Rührvorrichtung wird solange gerührt, bis das Wasser eine konstante Temperatur angenommen hat. Darauf wird gezündet und unter beständigem Rühren mit einer Lupe an einem in $1 / 50^{\circ} \mathrm{C}$. eingeteilten Thermometer die Erwärmung des Wassers abgelesen. Mit dem Thermometer, dessen Fehler genau bekannt sind, kann man auf $1 / 250^{\circ}$ genau ablesen. Ist z. B. der Kalorienfaktor ${ }^{1}$ ) des betreffenden Kalorimeters 1,3583, die Menge des verbrannten Kotes $=1,1058 \mathrm{~g}$ und betrug die Erwärmung des Wassers $3,925^{\circ}$, so ist der Brennwert für $1 \mathrm{~g}$ des betreffenden hotes

$$
=\frac{1,3583 \cdot 3,925}{1,1058} \text { Kalorien. }
$$

Davon ist der Brennwert des Zwirnsfadens in Abzug zu bringen, ferner der Brennwert des zur Abgrenzung benutzten Karmins. Derselbe beträgt nach meinen Untersuchungen 4,74 Kalorien für $1 \mathrm{~g}$ Karmin.

Jede Brennwertsbestimmung $\mathrm{mn} ß$ doppelt ausgeführt werden.

1) Der Kalorienfaktor des Kalorimeters ist diejenige Wärmemenge, welche nötig ist, um den im Kalorimeter vorhandenen einen Liter Wasser um $1^{\circ}$ C. zu erwärmen. Er muß für jeden Apparat besonders bestimmt werden. 
Der Tabelle, welche die Resultate unserer Untersuchungen wiedergibt, möchte ich folgende Bemerkungen vorausschicken:

Die in der Tabelle aufgeführten Kalorienzahlen, welche aus den Werten für Stickstoff, Fett und Kohlehydraten in der Nahrung und im Kot berechnet sind, sind als Rohkalorien aufzufassen. Wenn wir bei Ausnutzungsversuchen den Kalorienwert der eingeführten Versuchsnahrung mit dem des dieser Versuchsnahrung entstammenden Kotes vergleichen wollen, so dürfen wir, da wir doch den Gesamtkaloriengehalt der Nahrung der Resorption zuführen, zur Berechnung dieses Wertes nicht den physiologischen Nutzeffekt von Eiweiß, Fett und Kohlehydraten heranziehen, sondern müssen die wirklichen Brennwerte, wie sie im Kalorimeter gefunden werden, einsetzen. Für die Berechnung von Fett und Kohlehydraten ist dies ohne Belang; beide werden im Organismus bekanntlich zu Ende verbrannt, und man kann deshalb ihre Kalorienfaktoren 9,3 und 4,1 , die zugleich ihren physiologischen Nutzeffekt darstellen, ohne weiteres bei der Berechnung anwenden. Beim Eiweiß liegen die Verhältnisse insofern anders, als hier der physiologische Nutzeffekt, wie Rubner ${ }^{1}$ ) gezeigt hat, 4,1 ist, während der wirkliche Brennwert höher liegt. Stohmann²) hat aus verschiedenen Eiweißsorten einen Durchschnittswert von 5730,8 cal. berechnet. Berthelot ${ }^{3}$ ) fand als Mittel 5691 cal. Da man nun häufig in die Lage kommt, mit Durchschnittswerten zu rechnen, so nimmt Stohmann zu Berechnungen das Mittel aus diesen beiden Zahlen $=5711$ cal. oder 5,7 Kalorien. Rubner ${ }^{4}$ ) hat annähernd die gleichen Mittelwerte gefunden.

Wir haben demgemäß bei unseren Untersuchungen für die Berechnung der Eiweißbrennwerte die Zahl 5,7 als Kalorienfaktor benutzt. Daß diese Berechnung sehr genau ist, ergibt sich aus folgendem:

Unsere Versuchsnahrung, die Probediät von Schmidt und Strasburger, besteht aus:

i) $1 . c$.

2) l. c.

3) cit. nach Stohmann, l. c.

4) I. $\mathrm{c}$. 


$\begin{array}{rl}15 & 1 \text { Milch, } \\ 2 & \text { Eiern } \\ 100 & \text { g } \text { Zwieback } \\ 80 & \Rightarrow \text { Hafergrütze } \\ 50 & \Rightarrow \text { Butter } \\ 125 & \Rightarrow \text { Filet } \\ 190 & \Rightarrow \text { Kartoffeln. }\end{array}$

Dem entsprechen, nach Schwenkenbecher ${ }^{1}$ ) berechnet, 95 Eiweiß, 110,7 Fett, 191,3 Kohlehydrate. Aus der Berechnung des Eiweißes mit 5,7, des Fettes mit 9,3, der Kohlehydrate mit 4,1 resultieren 2355,7 Rohkalorien. Die direkte Verbrennung der Probediät im Kalorimeter liefert 2366,3 Kalorien, also eine sehr gute Übereinstimmung.

Wenn wir die Ausnutzung dieser Rohkalorienmenge im Darm feststellen wollen, so ist es, um vergleichbare Zahlen $\mathrm{zu}$ erhalten, nötig, das Koteiweiß ebenfalls mit 5,7 zu multiplizieren.

\section{Ergebnisse.}

Die Tabelle zeigt zunächst, daß zwischen den kalorimetrisch und chemisch bestimmten Brennwerten des Kotes in allen Fällen Differenzen bestehen, zum Teil erheblicher Art, und zwar sind die direkt gefundenen Brennwerte stets höher als die berechneten. Die absoluten Differenzen sind als Einzelzahlen und als Mittelzahlen höchst verschieden, ohne jede Regelmäßigkeit. Wenn man aber die Differenzen auf 100 direkt bestimmte Kalorien bezieht, so ist eine gewisse Übereinstimmung nicht zu verkennen. Die Differenzen (Mittelzahlen) schwanken dann zwischen 10 und 20 Kalorien mit Ausnahme der beiden mittleren Gruppen, wo sie noch niedriger liegen. Durchschnittlich resultieren also, wenn bei der Verbrennung von Faeces Erwachsener im Kalorimeter 100 Kalorien gefunden werden, ca. 12 Kalorien mehr, als man auf dem Wege der Elementaranalyse und Berechnung erhalten würde, die Anwendung der Probediät vorausgesetzt.

1) Die Nährwertberechnung tischfertiger Speisen. J. D. Marburg 1900. 
Hans Lohrisch,

\begin{tabular}{|c|c|c|c|c|c|c|c|}
\hline \multirow{2}{*}{$\mathrm{Nr}$. } & \multirow{2}{*}{ Diagnose } & \multirow{2}{*}{ Name } & \multicolumn{2}{|c|}{ Gesamtkotmenge } & \multicolumn{3}{|c|}{$\begin{array}{l}\text { Gesamtgehalt des trockenen } \\
\text { Kotes an: }\end{array}$} \\
\hline & & & feucht & trocken & $N$ & Fett & $\begin{array}{c}\text { Kohle- } \\
\text { hydraten } \\
\text { als Zucker } \\
\text { berechnet }\end{array}$ \\
\hline 1 & \multirow{5}{*}{$\begin{array}{l}\text { Normal- } \\
\text { kote }\end{array}$} & $\mathrm{Li}$ & $259, \check{5}$ & 60,3 & 3,52 & 12,93 & 2.9 \\
\hline 2 & & $\mathrm{Ka}$ & 219,0 & 62,0 & 3,09 & 13,6 & 1,4 \\
\hline 3 & & W & 270,0 & 55,6 & 2,32 & 14,8 & 1,44 \\
\hline 4 & & Schi & 225,0 & 51,6 & 2,94 & 10,4 & $2,1 \tilde{0}$ \\
\hline 5 & & St & 282,0 & 45,0 & 2,41 & 13,04 & $2,6 \check{~}$ \\
\hline \multicolumn{3}{|c|}{ Mittelwerte: } & 251,1 & 54,9 & 2,86 & 12,95 & 2,11 \\
\hline 6 & \multirow{3}{*}{$\begin{array}{l}\text { Gärungs- } \\
\text { Dyspepsie }\end{array}$} & $\mathrm{D}$ & 1070 & 159,9 & 9,82 & 35,48 & 5,32 \\
\hline 7 & & $\mathrm{Br}$ & 486,5 & 99,3 & 7,01 & 19,29 & 5,93 \\
\hline 8 & & $\mathrm{G}$ & 284,0 & 55,0 & 3,36 & 9,28 & 4,09 \\
\hline \multicolumn{3}{|c|}{ Mittelwerte: } & 613,5 & 104,7 & 6,73 & 21,35 & 5,11 \\
\hline 9 & \multirow{2}{*}{ Fettstïhle } & V & 1147 & 202 & 6,75 & 97,92 & 2,3 \\
\hline 10 & & $\mathrm{Ge}$ & 497 & 127 & 8,24 & 68,06 & 1,21 \\
\hline \multicolumn{3}{|c|}{ Mittelwerte: } & 822 & 165 & 7,49 & 82,99 & 1,75 \\
\hline 11 & \multirow{2}{*}{ Diarrhöen } & $\mathrm{K}$ & 593 & 108 & 6,91 & 32,92 & 3,0 \\
\hline 12 & & $\mathrm{~F}$ & 261 & 31 & 2,54 & 6,26 & 1,3 \\
\hline \multicolumn{3}{|c|}{ Mittelwerte: } & 427 & 70 & 4,72 & 19,59 & 2,2 \\
\hline 13 & $\begin{array}{l}\text { Chronische } \\
\text { habituelle }\end{array}$ & $\mathrm{Gr}$ & 130,0 & 37,0 & 1,58 & 8,74 & 2,1 \\
\hline 14 & Obstipation & M & 121,0 & 30,8 & 1,52 & 7,98 & 0,88 \\
\hline \multicolumn{3}{|c|}{ Mittelwerte: } & 125,0 & $\mathbf{3 3 , 9}$ & 1,55 & 8,36 & 1,49 \\
\hline 15 & \multirow{2}{*}{$\begin{array}{l}\text { Achylia } \\
\text { gastrica }\end{array}$} & $\mathrm{J}$ & 581 & 112,2 & 8,17 & 14,08 & 4,49 \\
\hline 16 & & $\mathrm{~L}$ & 474 & 85,6 & 4,43 & 19,93 & 5,2 \\
\hline \multicolumn{3}{|c|}{ Mittelwerte: } & 528 & 98,9 & 6,30 & 17,0 & 4,85 \\
\hline
\end{tabular}




\begin{tabular}{|c|c|c|c|c|c|}
\hline \multicolumn{3}{|c|}{ Gesamtkalorienmengen } & \multicolumn{2}{|c|}{$\begin{array}{c}\text { Differenz zwischen den } \\
\text { kalorimetrisch u. chemisch } \\
\text { bestimmten ausgeschie- } \\
\text { denen Kalorienmengen }\end{array}$} & \multirow{3}{*}{$\begin{array}{c}\text { Aus - } \\
\text { nützung: } \\
\text { Von } 100 \text { auf- } \\
\text { genommenen } \\
\text { Kalorien } \\
\text { werden im } \\
\text { Kot aus- } \\
\text { geschieden }\end{array}$} \\
\hline \multirow{2}{*}{$\begin{array}{c}\text { Auf- } \\
\text { genommen }\end{array}$} & \multicolumn{2}{|c|}{ im Kot ausgeschieden } & \multirow{2}{*}{$\begin{array}{l}\text { Absolute } \\
\text { Differenz }\end{array}$} & \multirow{2}{*}{\begin{tabular}{|c|} 
Differenz auf 100 \\
kalorimetrisch \\
bestiminte \\
Kaloricn \\
berechnet.
\end{tabular}} & \\
\hline & $\begin{array}{c}\text { kalorimetrisch } \\
\text { bestimmt }\end{array}$ & $\begin{array}{l}\text { chemisch } \\
\text { bestimmt }\end{array}$ & & & \\
\hline 7243,7 & 288,9 & 256,1 & $32,8^{\circ}$ & 11,4 & 3,9 \\
\hline 7000 & 268,3 & 242,3 & 26,0 & 9,7 & 3,8 \\
\hline 7000 & 241,1 & 227,1 & 14,0 & 5,8 & 3,4 \\
\hline 7099 & 251,7 & 210,6 & 41,1 & 16,4 & 3,5 \\
\hline 7099 & 262,6 & 218,0 & 46,6 & 17,0 & 3,7 \\
\hline 7088,3 & 262,5 & $\mathbf{2 3 0 , 8}$ & 32,1 & 12,1 & 3,7 \\
\hline 7000 & 800,9 & 702,3 & 98,6 & 12,3 & 11,4 \\
\hline 7469 & 496,3 & 453,3 & 43,0 & 8,5 & 6,6 \\
\hline 7000 & 283,1 & 222,7 & 60,4 & 21,3 & 4,0 \\
\hline 7156,3 & 526,8 & 459,4 & 67,3 & 14,0 & 7,3 \\
\hline 7000 & 1352,1 & 1160,4 & 191,7 & 14,2 & 19,3 \\
\hline $6933,0 ّ$ & 944,7 & 931,2 & 13,5 & 1,5 & 13,6 \\
\hline 6966,8 & 1148,4 & 1045,8 & 102,6 & 7,9 & 16,5 \\
\hline 7000 & 602,4 & 564,4 & 38,0 & 6,3 & 8,6 \\
\hline 1950 & $1 \check{5} 5, \check{b}$ & 152,9 & 2,6 & 1,7 & 7,9 \\
\hline 4475 & 378,9 & 358,7 & 20,3 & 4,0 & 8,3 \\
\hline 7099 & 176,8 & 145,5 & 31,3 & 17,7 & 2,4 \\
\hline 6994,6 & 163,6 & 131,6 & 32,0 & 19,6 & 2,3 \\
\hline 7046,8 & 170,2 & 135,7 & 31,6 & 18,7 & 2,35 \\
\hline 7099 & 499,0 & 439,1 & ŏ9,9 & 12,0 & 7,0 \\
\hline 7099 & 466,6 & 363,1 & 103,5 & 22,2 & 6,5 \\
\hline 7099 & 482,8 & 401,1 & 81,7 & 17,1 & 6,8 \\
\hline
\end{tabular}


Es fragt sich, worauf dieser Überschuß an Kalorien, der auf chemischem Wege nicht mitbestimmt wird, zu beziehen ist? Hierbei ist zunächst $\mathrm{zu}$ bedenken, daß bei der Analyse stets ein gewisser, wenn auch sehr kleiner Bruchteil von N, Fett und Kohlehydraten der Bestimmung entgeht. Dann werden z. B. Lecithin und Cholestearin, die durch Äther mit extrahiert werden, als Fett berechnet, während ihre eigentlichen Brennwerte höhere sind. Auch die Methoden der Kohlehydratbestimmung leiden an Ungenauigkeiten. Hiervon abgesehen können nur noch die im Kot außer N, Fett und Koblehydraten vorhandenen brennbaren Substanzen organischer und anorganischer Natur in Frage kommen. Von organischen Stoffen ist es einmal der Teil der ausgeschiedenen Zellulose, der bei der Kohlehydratbestimmung nicht mit in Zucker invertiert wird. Nach meinen Untersuchungen ist der Zellulosegehalt der Probediät so gering, daß sie für unsere Fälle bedeutungslos ist. Wichtiger sind schon die Gallenfarbstoffe, Gallensäuren und andere organische Säuren wie Oxalsäure, Milchsäure, Bernsteinsäure, die im Kot gelegentlich vorkommen. Endlich sind noch die anorganischen Substanzen des Kotes zu berücksichtigen. Die Kotasche enthält Chlornatrium, Chlorkalium, die Oxyde von Natrium, Kalium, Calcium, Magnesium, Eisen, ferner Phosphorsäure, Schwefelsäure, Kieselsäure, Sand, alles Substanzen, welche indes gar keinen oder nur minimalen Wärmewert besitzen.

Es geht aus dem Gesagten hervor, daß kalorimetrisch ausgeführte Ausnutzungsversuche, bei denen auf die Einzelwerte von N, Fett und Kohlehydraten nicht Wert gelegt wird, bei weitem exakter ausfallen als auf dem umständlichen Wege der chemischen Analyse.

Vom klinischen Standpunkt aus sind die Ergebnisse unserer Untersuchungen folgende: Wenn wir Vergleiche anstellen zwischen der Energiemenge der eingeführten Nahrung und der Menge von Kraft, die im Kot den Organismus verläßt, so erhalten wir dadurch Aufschluß darüber, in welchem. Umfang eine Resorption vom Darm aus stattgefunden hat. Es sind dies immerhin wertvolle Aufschlüsse, welche vor allen Dingen, wie auch Schloßmann ${ }^{1}$ )

1) l. c. 
an seinen Säuglingen gezeigt hat, für die Klinik brauchbar sind, indem sie uns anzeigen, entweder ob die Ernährung eine rationelle ist oder ob der Darm tadellos funktioniert. Bei vergleichenden Untersuchungen über die Ausnutzung der Nahrung in normalen und pathologischen Fällen kommt es natürlich darauf an, immer ein und dieselbe Nahrung zugrunde zu legen. Die Probediät von Schmidt und Strasburger ist hierzu außerordentlich geeignet, denn einmal stellt sie an die Darmtätigkeit geringe Ansprüche zufolge ihrer leichten Verdaulichkeit und kann erfahrungsgemäß von Gesunden und Darmkranken ohne Störungen genommen werden. Wir können also bei Anwendung dieser Diät in den meisten Fällen ohne weiteres auf die sekretorischen und resorptiven Fähigkeiten des Darmes schließen. Andererseits ist ihr Brennwert ein für allemal kalorimetrisch festgelegt, sodaß diesbezügliche Untersuchungen in Wegfall kommen.

Wenn wir nun in unseren Fällen den Teil der Nahrungsenergie, der im Kot wieder ausgeschieden wird, in Prozenten ausdrücken, so bekommen wir für die Normalkote übereinstimmende Werte von 3,4-3,9\% (s. Tabelle), d. h. die durchschnittliche Kraftmenge, die ein Erwachsener mit normal funktionierendem Darm bei Anwendung der Probediät im Kot verlieren darf, beträgt 3,7\% der eingeführten Kalorienmenge. Der Reinwert der Probediät würde sich demnach auf $96,3 \%$ belaufen. Schloßmann ${ }^{1}$ ) hat bei gleichmäßig mit Milch ernährten Säuglingen die Grenze der normalen Kalorienausfuhr durch den Kot zu 10\% der aufgenommenen Kalorienmenge angegeben. Unter Zugrundelegung des Normalwertes 3,7\% ist es außerordentlich einfach, auch in pathologischen Fällen ein sicheres Urteil über die Funktion des Darms zu erhalten. Man hat nur den Brennwert des Kotes mit dem konstanten Brennwert der Probediät $\mathrm{zu}$ vergleichen, um $\mathrm{zu}$ erkennen, wieviel von der genossenen Nahrung resorbiert wird und ob die Resorption genügend ist. So finden wir bei unsern pathologischen Fällen einen zum Teil sehr hohen Prozentgehalt an Energie im Kot, in jedem Falle höher als im Normalkot. Eine bemerkenswerte Ausnahme machen die Fälle von chronischer habitueller Ob-

i) $1 . \mathrm{c}$. 
stipation; hier wird die Nahrung so vorzüglich ausgenutzt, daß der Verlust durch den Kot nur 2,35\% beträgt. ${ }^{1}$ )

In dieser Weise für Ausnutzungsversuche angewendet, erscheint die Kalorimetrie als eine recht brauchbare und einfache Methode, um sich über die Verhältnisse der Darmtätigkeit in normalen und pathologischen Fällen schnell und sicher zu orientieren. Auch für Untersuchungen des Kraftwechsels würde die Kalorimetrie wertvoll sein; man hätte hier außer dem Brennwert des Kotes noch den des Harns zu bestimmen, eine Untersuchung, die nach der von Schloßmann ${ }^{2}$ ) angegebenen Methode einfach und exakt ausführbar ist.

Alles in allem ist die Kalorimetrie, wenn man sie in dieser Weise für klinische Zwecke nutzbar macht, eine sehr wertvolle, genaue und wenig zeitraubende Untersuchungsmethode, deren Schwierigkeiten ihren zahlreichen Vorteilen gegenüber völlig in den Hintergrund treten.

Zum Schlusse erlaube ich mir, Herrn Prof. Dr. Schmidt für die Anregung zu dieser Arbeit und die Überlassung einer großen Anzahl von Faeces meinen verbindlichsten Dank auszusprechen.

1) Lohrisch, Die Ursachen der chronischen habituellen Obstipation im Lichte systematischer Ausnutzungsversuche. Deutsches Archiv für klin. Medizin, Bd. 79.

2) Zur Technik der kalorimetrischen Untersuchungsmethoden. Diese Zeitschrift, 1903, Bd. XXXVII, Heft 4 . 